\title{
High Prevalence and Incidence of Asymptomatic Sexually Transmitted Infections During Pregnancy and Postdelivery in KwaZulu Natal, South Africa
}

\author{
Dhayendre Moodley, PhD, * Prashini Moodley, PhD, † Motshedisi Sebitloane, MD, * \\ Deepak Soowamber, BBusSci,f Heather Luz McNaughton-Reyes, PhD, $\$$ \\ Allison K. Groves, PhD, I and Suzanne Maman, PhD
}

\begin{abstract}
Background: We report the prevalence and incidence of 3 treatable sexually transmitted pathogens (Neiserria gonorrhoeae, Chlamydia trachomatis, and Trichomonas vaginalis) in women who were HIV infected or at high risk for HIV infection, in pregnancy and postpartum, respectively.

Method: Vulvovaginal specimens collected at the first antenatal visit and again at 14 weeks postpartum were tested for $N$. gonorrhoeae, $C$. trachomatis, and T. vaginalis in the laboratory. Women were routinely tested for HIV-1 with a point-of-care test.
\end{abstract}

Results: Among 1480 women, 32.3\% (95\% confidence interval, 29.9-34.7) tested positive for any of the sexually transmitted infections (STIs) in pregnancy and 19.2\% (95\% confidence interval, 16.9-21.5) were positive when retested 14 weeks postpartum (incidence rate, 79.2 per 100 person-years). The prevalence of $N$. gonorrhoeae and $T$. vaginalis infections in pregnancy and the incidence rate of any STI at 14 weeks postpartum were significantly higher in HIV-1-infected women $(P<0.0001$ amd $P=0.0079)$. More than $50 \%$ of $N$. gonorrhoeae, $T$. vaginalis, and C. trachomatis infections in pregnancy were asymptomatic.

Conclusions: The high prevalence of asymptomatic STIs in pregnancy is compelling evidence that demands the development and validation of pointof-care tests for STIs be expedited. In addition, the high incidence of STIs 3 months postpartum suggests that women in this study setting resume unprotected sexual intercourse soon after delivery.

$A^{r}$ recent World Health Organization report estimated that 500 million adults between the ages of 15 and 49 years acquire 1 of 4 curable sexually transmitted bacterial infections (Neiserria gonorrhoeae, Chlamydia trachomatis, Treponema pallidum, and Trichomonas vaginalis) each year. ${ }^{1}$ In 2008 alone, 93 million new cases of these sexually transmitted infections (STIs) were reported in Africa. Almost half the cases were reported in women, with 4.3 million cases attributable to $C$. trachomatis, 9.6 million to $N$. gonorrhoeae, 1.6 million to T. pallidum, and 28.1 million cases to $T$. vaginalis.

From the *Department of Obstetrics and Gynaecology, University of KwaZuluNatal, Durban, South Africa; †Department of Infection, Prevention and Control, University of KwaZulu-Natal, Durban, South Africa; \$CAPRISA, Durban, South Africa; §University of North Carolina at Chapel Hill, Chapel Hill, NC; and $\uparrow$ Center on Health, Risk and Society, American University, Washington, DC

Funding: This work was supported by the Eunice Kennedy Shriver National Institute of Child Health and Human Development (Grant No. R01HD050134) and The Open Society Institute and Elton John Foundation (Grant No. 20020472/20030878).

Conflict of interest: None declared.

Correspondence: Dhayendre Moodley, PhD, Department of Obstetrics and Gynaecology, University of KwaZulu-Natal, Durban, South Africa. E-mail: moodleyd1@ukzn.ac.za.

Received for publication July 25, 2014, and accepted October 22, 2014. DOI: 10.1097/OLQ.0000000000000219

Copyright (C) 2014 American Sexually Transmitted Diseases Association All rights reserved.
Several studies have described the sequelae of STIs in women to include spontaneous abortion, preterm labor, infection in neonates, and pelvic inflammatory disease associated with ectopic pregnancies and infertility. ${ }^{2,3}$

More importantly, with particular reference to populations at high risk for HIV infection, an association between the classical STIs and HIV has triggered the need for early detection and prompt treatment of STIs. ${ }^{4,5}$ These cohort and laboratory-based studies, together with hypothetical models, have demonstrated plausible mechanisms of how the bacterial causes of STIs could facilitate the receptive transmission of their viral counterpart viz. HIV.

Hypothetically, the immediate and complete treatment of curable STIs detected in women could significantly reduce the risk for HIV infection. The syndromic management of STIs based on clinical, epidemiological, and microbiological evidence has been touted as the most feasible and cost-effective approach to reducing the prevalence of STIs and is strongly recommended by the World Health Organization for use in resource-limited settings. ${ }^{6}$ This multifaceted approach adopted in South Africa in 1995 includes treatment of symptomatic infection in addition to risk reduction counseling, contact tracing, and provision of condoms. ${ }^{7}$ However, the syndromic management strategy is not without its limitations. Several studies validating the symptom-based diagnostic approach against laboratory diagnosis have already reported several missed opportunities for treatment and control in the general adult population. ${ }^{8,9}$ There are few studies that have described the burden of STI disease in pregnancy, and only one other study has reported the incidence of STIs in postpartum women. ${ }^{10}$

In a randomized controlled trial designed to evaluate an HIV counseling intervention (SAHAPS study) conducted at a primary health care clinic in South Africa, specific STIs and inconsistent condom use in pregnancy and postdelivery served as biological and behavioral markers for risk reduction respectively. ${ }^{11}$ The primary analysis revealed no effect of enhanced counseling on incident STIs. ${ }^{11}$

We report the prevalence of STIs in this cohort of pregnant women and the incidence at 14 weeks postdelivery, with the assumption that all STIs detected in pregnancy were treated and had resolved before delivery. We further explore the association between these curable STIs and HIV and evaluate the validity of syndromic management in the detection and prompt treatment of the classical STIs at these critical time points.

\section{METHODS}

Between May 2008 and June 2010, antenatal attendees at a primary health clinic in Durban, who consented to participate in the SAHAPS study, were tested for HIV and the other STIs (N. gonorrhoeae, C. trachomatis, and $T$. vaginalis) at the first antenatal visit in pregnancy (baseline) and subsequently at 14 weeks postpartum. Participant recruitment, eligibility criteria, randomization, and retention strategy are described in greater detail in 
the primary article. ${ }^{11}$ Briefly, for the purpose of this secondary data analysis, we included 1480 pregnant women enrolled in the SAHAPS study. To be eligible for enrollment in the SAHAPS study, women had to have a primary intimate partner for the past 6 months.

\section{Procedures}

Study nurses provided antenatal care to all study participants according to the South African Antenatal Care guidelines. ${ }^{12}$ The antenatal and 14 week postpartum assessments included a collection of a vulvovaginal specimen. During the collection of these vulvovaginal specimens, nurses noted the presence or absence of an abnormal vaginal discharge. In addition, the syndromic management protocol was applied, and women were asked if they thought they had an abnormal vaginal discharge. The specimen swabs were temporarily refrigerated at the clinic and transported within 6 hours to the STI diagnostic laboratory in the Department of Infection Control, University of KwaZulu-Natal.

\section{Detection of N. gonorrhoeae, C. trachomatis, and $T$. vaginalis}

The swabs were processed for $N$. gonorrhoeae, $C$. trachomatis, and $T$. vaginalis using the BD Probetec ET Amplified DNA Assay (Becton Dickinson, Sparks, MD) using Strand Displacement technology to detect $N$. gonorrhoeae, C. trachomatis, and an in-house validated polymerase chain reaction assay using the primer set SarR and SarF specific for T. vaginalis. ${ }^{13}$

\section{Detection of HIV-1}

Serological testing for HIV-1 was performed at the clinic using a point of care (POC) testing algorithm. At their first antenatal visit, women were provided HIV pretest counseling and tested for HIV-1 according to the HIV testing algorithm used in the antenatal clinic. A participant provided a blood specimen from a finger prick for a POC HIV test (Determine: Abbott Laboratories, Abbott Park, IL). If a reactive result was obtained with the Determine rapid HIV test, the participant's HIV status was confirmed with a second POC test (Smart Check: World Diagnostic Inc, Miami Lakes, FL). Women who tested negative at the baseline visit were routinely retested at 34 to 36 weeks' gestation using the same testing algorithm. Nurses conducted posttest counseling sessions with women. Details of the content of posttest counseling are provided in the primary article. ${ }^{11}$

In compliance with the local syndromic management protocol, women reporting or presenting with symptoms suggestive of a sexually transmitted disease were initiated on treatment on the same day. Results from the laboratory diagnosis were available within 2 to 3 weeks, and women who were asymptomatic but tested positive by laboratory diagnosis were contacted and asked to return to the clinic at their earliest convenience for initiation of treatment. Every effort was made to ensure initiation of treatment as soon as possible.

\section{Statistical Analysis}

The data specific for this secondary analysis were selectively extracted from the "medical extraction form" database that housed the details and results of all laboratory investigations, as well as a single variable that described an infection as "symptomatic" or "asymptomatic" based on the presence of an abnormal vaginal discharge, as observed by the examining nurse or a verbal report by the participant. Prevalence was calculated as the percentage of women with the STI and 95\% confidence intervals (CIs) were calculated for these percentages, using the formulas for calculating CIs for proportions. $P$ values were calculated using Fisher exact test. Incidence rates, with $95 \%$ CIs, were calculated as the number of new cases of STIs over the total number of patient years at risk. Risk ratios were also calculated using a logbinomial regression. All statistical analyses were done with SAS 9.3 (SAS Institute Inc, Cary, NC).

The study was approved by the institutional review boards of the University of KwaZulu-Natal and the University of North Carolina at Chapel Hill. A written informed consent was obtained from all women who participated in the main study.

\section{RESULTS}

\section{Sample Recruitment, Retention, and Laboratory Diagnosis}

A total of 1480 pregnant women who completed a baseline assessment before being randomized to 1 of 2 study arms in the SAHAPS study were included in this analysis. Data were missing for $21(1.4 \%)$ women who were therefore excluded from the analysis at baseline. The number of women who tested for each of the 3 curable STIs in pregnancy and subsequently at the 14 -week postpartum visit is illustrated in Figure 1. At the primary outcome visit (14 weeks postdelivery), 1183 (79.9\%) women returned for followup and laboratory results were available for 1124 women who tested for $N$. gonorrhoeae, C. trachomatis, and T. vaginalis.

\section{Baseline Characteristics}

As previously reported in the primary outcome article, the average gestational age at booking was 24 weeks, 37\% (554/1480) were first-time pregnancies and $25.9 \%(384 / 1480)$ were living with their partner. ${ }^{11}$ Inconsistent condom use was common, with $68.9 \%$ $(1020 / 1480)$ of participants at baseline reported having had sex without a condom in the past 30 days and fewer women (14\%) reported inconsistent condom use at 14 weeks postdelivery. We did not collect information on condom use during the different stages of pregnancy or between the baseline assessment and delivery.

\section{Prevalence and Incidence of STIs in Pregnancy and 14 Weeks Postdelivery}

The percentage of pregnant women testing positive for an STI excluding HIV was $32.3 \%$ (95\% CI, 29.9-34.7) at baseline and $19.2 \%(95 \% \mathrm{CI}, 16.9-21.5)$ at 14 weeks postdelivery. C. trachomatis and $T$. vaginalis were the most common pathogens detected in pregnancy: $17.8 \%$ and $15.3 \%$, respectively. $N$. gonorrhoeae was detected in $6.4 \%$ of the women (Table 1 ).

When women were tested again at 14 weeks postdelivery, $C$. trachomatis and $T$. vaginalis remained the most commonly detected pathogens but at a lower prevalence of $10.1 \%$ and $8.9 \%$, respectively. $N$. gonorrhoeae was detected in $2.9 \%$ of the women postdelivery.

In total, among 1124 women tested at 14 weeks postdelivery, there were $216(19.2 \%)$ women with an STI at 14 weeks postdelivery. The STI results for 1 woman among the 216 cases were inconclusive in pregnancy and were not reported as new infections at 14 weeks. Overall, there were 86 new STIs at 14 weeks postdelivery, among 986 women who were uninfected at screening in pregnancy, with an incidence rate of 43.8 per 100 person-years (95\% CI, 35.1-54.1; Table 2). Of 337 women who had an STI at 


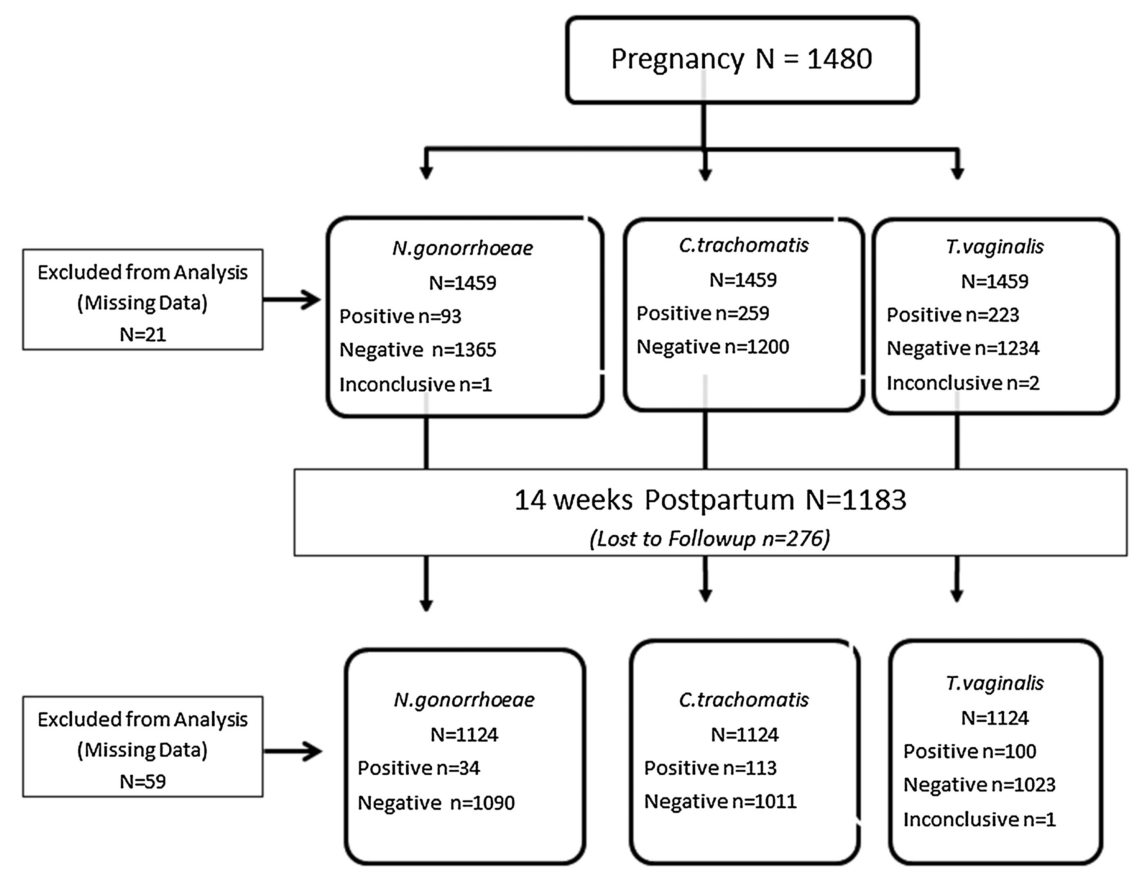

Figure 1. Flow diagram illustrating the number of women tested for each of the STIs at baseline (pregnancy) and subsequently at 14 weeks postdelivery.

baseline and retested at 14 weeks postdelivery, 129 (38.3\%) also had a detectable STI at 14 weeks postdelivery $(21 \%$. gonorrhoeae, $27 \%$ T. vaginalis, and $30 \%$ C. trachomatis). Given that all women with an STI at baseline were treated during pregnancy, the 216 infections detected at 14 weeks postdelivery among 1124 women could also be considered as new infections; incidence rate 79.2 per 100 person-years (95\% CI, 69.0-90.5).

\section{Prevalence and Incidence of STI in Association With HIV}

A total of 571 of the 1480 pregnant women tested positive for HIV at their first antenatal visit (HIV prevalence, 38.58\%; 95\% CI, 36.10-41.12). Among 410 seronegative women, the overall incidence rate of HIV at 14 weeks was $5.49 / 100$ person-years $(95 \%$ CI, 2.01-11.95). In pregnancy, there were no differences in the prevalence estimates of any STI stratified by HIV status; however, the prevalences of gonorrhea $(9.8 \%$ vs. $4.4 \%)$ and trichomoniasis $(22.6 \%$ vs. $10.5 \%)$ were significantly higher in the HIV-positive women $(P<0.0001)$. Overall, the incidence rate of any STI at 14 weeks postdelivery was also significantly higher among women who tested HIV positive in pregnancy (58.0 per 100 person-years vs. 36.4 per 100 person-years; $P=0.0079$ ). More specifically, the incidence of gonorrhea and that of trichomoniasis were 5.5 times and 1.8 times higher in the HIV-positive women, respectively (Table 3 ).

\section{Asymptomatic STIs and Syndromic Management}

Among the 470 women who tested positive for any of the 3 STIs, $206(43.8 \%)$ were symptomatic and were treated syndromically ( $N$. gonorrhoeae $47.3 \%$, T. vaginalis $47.1 \%$, and C. trachomatis $42.9 \%)$ Furthermore, only $26(12 \%)$ of 216 women with an STI postdelivery were symptomatic, although the specificity of the syndromic approach was much higher postdelivery (specificity $96.9 \%$, negative predictive value $82.2 \%$ ) as compared with pregnancy (specificity $68.7 \%$, negative predictive value $71.9 \%$ ).

\section{DISCUSSION}

In this data analysis of a cohort of 1480 women enrolled in pregnancy at a primary health care clinic with an antenatal HIV prevalence of $38 \%, 1(32.3 \%)$ in 3 women tested positive for at least one of the curable bacterial STIs ( $N$. gonorrhoeae, C. trachomatis, or $T$. vaginalis) at the first antenatal visit and 1 $(19.2 \%)$ in 5 women tested positive at 14 weeks postdelivery. Considering the limited number of STI cohort studies conducted among pregnant women, this is the first report of incident STIs detected within 3 months postpartum in a population with a high HIV antenatal seroprevalence. In addition, more than $50 \%$ of women with an STI in pregnancy and more than $80 \%$ with an STI postdelivery were asymptomatic.

TABLE 1. Prevalence of STIs at Baseline (Pregnancy) and the Percentage of STIs at 14 Weeks Postdelivery

\begin{tabular}{|c|c|c|c|c|c|c|}
\hline \multirow{3}{*}{$\frac{\text { STI }}{\text { N. gonorrhoeae }}$} & \multicolumn{3}{|c|}{ Baseline $(n=1480)$} & \multicolumn{3}{|c|}{ Postdelivery $(n=1183)$} \\
\hline & \multirow{2}{*}{$\frac{\%(n / \mathbf{N})}{6.4(93 / 1459)}$} & \multicolumn{2}{|c|}{$95 \% \mathrm{CI}$} & \multirow{2}{*}{$\frac{\%(\mathbf{n} / \mathbf{N})}{2.9(33 / 1124)}$} & \multicolumn{2}{|c|}{$95 \%$ CI } \\
\hline & & 5.12 & 7.63 & & 1.95 & 3.93 \\
\hline T. vaginalis & $15.3(223 / 1459)$ & 13.46 & 17.15 & $8.9(100 / 1124)$ & 7.23 & 10.56 \\
\hline C. trachomatis & $17.8(259 / 1459)$ & 15.79 & 19.71 & $10.1(113 / 1124)$ & 8.30 & 11.81 \\
\hline Any STI & $32.2(470 / 1459)$ & 29.88 & 34.68 & $19.2(216 / 1124)$ & 16.91 & 21.52 \\
\hline
\end{tabular}


TABLE 2. Incidence of STIs at 14 Weeks Postdelivery in Women Who Were Uninfected in Pregnancy and in All Women Assuming Complete Resolution of STIs Detected in Pregnancy

\begin{tabular}{|c|c|c|c|c|c|}
\hline \multirow{4}{*}{$\begin{array}{l}\text { STI } \\
\text { N. gonorrhoeae } \\
\text { T. vaginalis }\end{array}$} & \multicolumn{2}{|c|}{ Women Uninfected in Pregnancy } & \multicolumn{3}{|c|}{ All Women (Women Infected and Uninfected in Pregnancy)* } \\
\hline & \multirow{2}{*}{$\frac{\text { Incidence Rate (Per 100 Person-Years) }}{7.2(20 / 279)}$} & $95 \% \mathrm{CI}$ & \multirow{2}{*}{$\frac{\text { Incidence Rate (Per 100 Patient-Years) }}{11.1(33 / 297)}$} & \multicolumn{2}{|c|}{$95 \%$ CI } \\
\hline & & $4.4 \quad 11.1$ & & 7.65 & 15.60 \\
\hline & $22.9(57 / 249)$ & $17.3 \quad 29.6$ & $34.7(100 / 288)$ & 28.22 & 42.18 \\
\hline C. trachomatis & $22.9(55 / 240)$ & $17.3 \quad 29.8$ & $39.4(113 / 287)$ & 32.49 & 47.40 \\
\hline Any STI & $43.8(86 / 196)$ & $35.0 \quad 54.1$ & $79.2(216 / 273)$ & 68.97 & 90.45 \\
\hline
\end{tabular}

*Assuming $100 \%$ cure rate of STIs treated in pregnancy.

The only empirical studies conducted among pregnant women in South Africa have described lower prevalence estimates than those reported in our study. Locally, in a small study of pregnant women $(\mathrm{n}=168)$, the prevalence of chlamydia and gonorrhea in 1994 was almost half of what is reported in the current study. ${ }^{14}$ Our prevalence estimate of STIs in pregnant women enrolled between 2008 and 2010 was similar to that reported among HIVuninfected nonpregnant women with multiple partners between 2004 and $2006 .{ }^{15}$ In this study, Mlisana et al. ${ }^{15}$ reported T. vaginalis as the single most common infection in 242 nonpregnant highrisk HIV-uninfected women, whereas we reported $C$. trachomatis in addition to $T$. vaginalis as the more common infections in both HIV-infected and HIV-uninfected women during pregnancy $(\mathrm{n}=1456)$ and postdelivery $(\mathrm{n}=1124)$. Of note, the antenatal HIV seroprevalence in South Africa in 1994 was 7.6\% and subsequently increased exponentially to reach an epidemic plateau in $2004(29.5 \%) .{ }^{16}$ The temporal association between the increased prevalence of HIV and STIs in pregnancy alone is an indication of the high sexual behavioral risk that has been grossly understudied in pregnancy and demands more attention in respect of HIV prevention, risk reduction counseling, and management of STIs.

Our findings raise many concerns related to diagnosis, management, and prevention of STIs in women during and after pregnancy. Our most significant findings were the higher prevalence of gonorrhea (9.6\% vs. $4.4 \%)$ and trichomoniasis $(22.9 \%$ vs. $6.5 \%$ ) among HIV-positive women and the significantly higher incidence rate of any STI at 14 weeks postdelivery among women who tested HIV positive in pregnancy (59.8 per 100 person-years vs. 37.2 per 100 person-years). More specifically, the incidences of gonorrhea and trichomoniasis were 5.6 times and 1.9 times higher in the HIV-positive women, respectively. Our findings reflect that HIV-infected women are more likely predisposed to other STIs. The higher incidence of STIs among HIV positive women in our study is reflective of higher behavioral risk in this cohort. These findings are consistent with other studies that reported similar trends in behavioral risk among other high-risk population groups. ${ }^{17-19}$ HIV-infected individuals were more likely to report unprotected sex than their HIV-uninfected counterparts.

The high incidence of STIs 3 months postpartum also suggests that women in this study setting resume unprotected sexual intercourse soon after delivery. The increased susceptibility to other STIs in HIV-infected women has serious implications for maternal and child health and certainly demands a comprehensive risk reduction strategy similar to that proposed for HIV-uninfected women. Sexually transmitted infections and other coinfections through evoking mucosal inflammatory responses are known to amplify HIV shedding in cervical fluids, which in turn could increase risk of mother-to-child transmission of HIV and rapid HIV disease progression in the mother. ${ }^{20-23}$

Our other significant finding that is consistent with several previous studies is critically persuasive for South Africa and other countries to reconsider their syndromic approach to managing STIs in pregnancy, particularly in settings with a high HIV prevalence. Treatment of symptomatic STIs, partner tracing, and advocacy for consistent and correct use of condoms seem to be the most affordable and feasible option in resource-limited countries. Our findings and that of other recent studies, however, question the validity of this approach based on the large proportion of asymptomatic STIs prevalent in pregnant and other high-risk population groups. ${ }^{15,24-27}$ When extrapolating our findings to all primary health clinics using the syndromic approach to treating STIs in women, it would appear that an estimated $50 \%$ of pregnant women with 1 or more STIs and asymptomatic will go untreated if the syndromic approach is applied. Postdelivery, an estimated $80 \%$ of the women who are asymptomatic but infected with 1 or more STIs would be missed. High estrogen levels in pregnancy are known to be associated with increased production of vaginal discharge and women are often unable to distinguish between abnormal discharge associated with STIs and normal pregnancyinduced vaginal discharge. Although the specificity of the syndromic approach is lower in pregnancy when compared with postpregnancy, women with asymptomatic STIs are more likely to be treated in pregnancy as compared with postpregnancy because of the overreporting of "abnormal" vaginal discharge during pregnancy.

Although our study provides compelling evidence for prompt diagnosis of STIs in pregnancy, without POC tests, we have no other feasible and affordable alternative to the syndromic management approach. Although POC tests are being developed and validated, the selective use of the Cepheid GeneXpert currently being used

TABLE 3. Incidence of STIs in HIV-Positive and HIV-Negative Women Postdelivery (14 Weeks)

\begin{tabular}{|c|c|c|c|c|c|c|}
\hline STI & $\begin{array}{c}\text { HIV-Positive Women, } \\
\text { Incidence Rate of STI } \\
\text { Per } 100 \text { Person-Years (n/py) }\end{array}$ & $95 \%$ CI & $\begin{array}{c}\text { HIV-Negative Women, } \\
\text { Incidence Rate of STI } \\
\text { Per } 100 \text { Person Year (n/py) }\end{array}$ & 95\% CI & $\begin{array}{c}\text { Incidence } \\
\text { Rate Ratio }\end{array}$ & $P$ \\
\hline N. gonorrhoeae & $15.6(15 / 95.986)$ & $8.75-25.77$ & $2.8(5 / 178.145)$ & $0.91-6.55$ & 5.47 & 0.001 \\
\hline T. vaginalis & $32.9(27 / 82.025)$ & $21.69-47.89$ & $17.6(29 / 164.452)$ & $11.8-25.33$ & 1.83 & 0.02 \\
\hline C. trachomatis & $25.6(21 / 82.025)$ & $15.85-39.14$ & $20.8(32 / 153.847)$ & $14.2-29.36$ & 1.22 & 0.44 \\
\hline Any STI & $58.0(36 / 62.022)$ & $40.65-80.36$ & $36.4(48 / 131.830)$ & $26.9-48.28$ & 1.55 & 0.03 \\
\hline
\end{tabular}


to detect tuberculosis in South Africa could be explored for highrisk pregnant including women who are HIV infected. ${ }^{28-30}$ We could also improve our existing prevention, diagnostic, and treatment strategy for STI management. We would recommend the following to be practiced at multiple visits between registration for antenatal care and postdelivery: (i) client-centered risk reduction counseling, (ii) history taking and an extensive personal interview to determine sexual risk and establish signs and symptoms of an STI, (iii) an uninterrupted supply of good quality condoms for correct and consistent use, (iv) more effort at partner notification and referral for treatment, and most importantly (v) repeat screening using the syndromic approach in the third trimester of pregnancy or before delivery and again at 3 months postdelivery.

Our study is not without limitations. Our $80 \%$ retention at the 14-week postdelivery visit could selectively exclude women at high risk, hence providing an underestimate of the STI incidence. Although all women with a laboratory diagnosis of STIs were treated within 2 to 3 weeks or at the next antenatal visit, we did not adjust for these variabilities and treatment adherence when calculating STI incidence at 14 weeks postdelivery.

\section{REFERENCES}

1. World Health Organisation. Global Incidence and Prevalence of Selected Curable Sexually Transmitted Infections - 2008. Geneva: World Health Organisation, 2012. Available at: http://www.who.int/ reproductivehealth/publications/rtis/stiestimates/en/. Accessed July 2, 2014.

2. Schulz KF, Cates W, O'Mara PR. Pregnancy loss, infant death, suffering: Legacy of syphilis and gonorrhoeae in Africa. Genitourin Med 1987; 63:320-325.

3. Moodley P, Sturm AW. Sexually transmitted infections, adverse pregnancy outcome and neonatal infection. Semin Neonatal 2000; 5:255-269.

4. Sexton J, Garnett G, Rottingen J-A. Metaanalysis and metaregression in interpreting study variability in the impact of sexually transmitted diseases on susceptibility to HIV infection. J Sex Transm Dis 2005; 32:351-357.

5. Rottingen JA, Cameron DW, Garnett GP. A systematic review of the epidemiologic interactions between classic sexually transmitted diseases and HIV: How much really is known? Sex Transm Dis 2001; 28:579-597.

6. World Health Organization. Guidelines for the management of sexually transmitted infections. ISBN 924 1546263. Available at: http://www.who.int/hiv/pub/sti/pub6/en/. Accessed February 1, 2004

7. National Department of Health. First Line Comprehensive Management and Control of Sexually Transmitted Infections (STIs): Protocol for the Management of a Person With a Sexually Transmitted Infection According to the Essential Drug List. Pretoria: National Department of Health, 2008:1-18.

8. Ghebremichael M. The Syndromic versus laboratory diagnosis of sexually transmitted infections in resource-limited settings. ISRN AIDS 2014; 103452. doi: 10.1155/2014/103452.

9. Liu H, Jamison D, Li X, et al. Is syndromic management better than the current approach for treatment of STDs in China? Evaluation of the cost-effectiveness of syndromic management for male STD patients. Sex Transm Dis 2003; 30:327-330.

10. Wheeler R, Earnshaw VA, Kershaw T, et al. Postpartum sexually transmitted disease refining our understanding of the population at risk. Sex Transm Dis 2012; 39:509-513.

11. Maman S, Moodley D, McNaughton-Reyes L, et al. Efficacy of enhanced HIV counseling for risk reduction during pregnancy and in the postpartum period among HIV-negative and HIV-positive women: Results from a randomized controlled trial. PLoS One 2014; 9:e97092. doi:10.1371/journal.pone.0097092.
12. Department of Health, South Africa. Guidelines for Maternity Care in South Africa: A Manual for Clinics, Community Health Centers and District Hospitals. 3rd ed. Pretoria: Department of Health, 2007.

13. Sturm PDJ, Connolly C, Khan N, et al. Vaginal tampons as specimen collection device for the molecular diagnosis of non-ulcerative sexually transmitted infections in antenatal clinic attendees. Int J STD AIDS 2004; 15:94. DOI:10.1258/095646204322764271.

14. Govender L, Hoosen AA, Moodley J, et al. Bacterial vaginosis and associated infections in pregnancy. Int J Gynaecol Obstet 1996; 55:23-28.

15. Mlisana K, Naicker N, Werner L, et al. Symptomatic vaginal discharge is a poor predictor of sexually transmitted infections and genital tract inflammation in high-risk women in South Africa. J Infect Dis 2012; 206:6-14.

16. The National Antenatal Sentinel HIV and Syphilis Prevalence Survey, South Africa. National Department of Health, 2011.

17. Djoba Siawaya JF. Chlamydia trachomatis, human immunodeficiency virus (HIV) distribution and sexual behaviours across gender and age group in an African setting. PLoS One 2014; 9:e90174 doi:10.1371/journal.pone.0090174.

18. Landes M, Thorne C, Barlow P, et al. Prevalence of sexually transmitted infections in HIV-1 infected pregnant women in Europe. Eur J Epidemiol 2007; 22:925-36. Epub 2007 Oct 10.PMID: 17926135.

19. Marks G, Crepaz N, Senterfitt JW, et al. Meta-analysis of high-risk sexual behavior in persons aware and unaware they are infected with HIV in the United States: Implications for HIV prevention programs. J Acquir Immune Defic Syndr 2005; 39:446-453.

20. Apalata T, Longo-Mbenza B, Sturm AW, et al. Factors associated with symptomatic vulvovaginal candidiasis: A study among women attending a primary healthcare clinic in Kwazulu-Natal, South Africa. Ann Med Health Sci Res 2014; 4:410-416 doi: 10.4103/2141-9248.133470. PMCID: PMC4071743.

21. Herold BC, Keller MJ, Shi Q, et al. Plasma and mucosal HIV viral loads are associated with genital tract inflammation in HIV-infected women. J Acquir Immune Defic Syndr 2013; 63:485-493. doi: 10.1097/QAI.0b013e3182961cfc.

22. Tubiana R, Le Chenadec J, Rouzioux C, et al. Factors associated with mother-to-child transmission of HIV-1 despite a maternal viral load $<500$ copies/ml at delivery: A case-control study nested in the French perinatal cohort (EPF-ANRS CO1). Clin Infect Dis 2010; 50:585-596. doi: $10.1086 / 650005$.

23. Mofenson LM, Lambert JS, Stiehm ER, et al. Risk factors for perinatal transmission of human immunodeficiency virus type 1 in women treated with zidovudine. Pediatric AIDS Clinical Trials Group Study 185 Team. N Engl J Med 1999; 341:385-393.

24. Otieno FO, Ndivo R, Oswago S, et al. Evaluation of syndromic management of sexually transmitted infections within the Kisumu Incidence Cohort Study. Int J STD AIDS 2014; 25:851-859.

25. Romoren M, Sundby J, Velauthapillai M, et al. Chlamydia and gonorrhoeae in pregnant Batswana women: Time to discard the syndromic approach? BMC Infect Dis 2007; 7:27.

26. Wilkinson D, Abdool Karim SS, Harrison A, et al. Unrecognised sexually transmitted infections in rural South African Women: A hidden epidemic. Bull World Health Organ 1999; 77:22-28.

27. White RG, Moodley P, McGrath N, et al. Low effectiveness of syndromic treatment services for curable sexually transmitted infections in rural South Africa. Sex Transm Infect 2008; 84:528-534. doi: 10.1136/sti.2008.032011. Epub 2008 Aug 15.

28. Gaydos C, Hardick J. Point of care diagnostics for sexually transmitted infections: Perspectives and advances. Expert Rev Anti Infect Ther 2014; 12:657-672. doi:10.1586/14787210.2014.880651.

29. Natoli L, Maher L, Shephard M, et al. Point-of-care testing for chlamydia and gonorrhoeae: Implications for clinical practice. PLoS One 2014; 9:e100518. doi:10.1371/journal.pone.0100518.

30. Parcell BJ, Ratnayake L, Kaminski G, et al. Value of repeat testing using Cepheid GeneXpert CT/NG for indeterminate PCR results when diagnosing Chlamydia trachomatis and Neisseria gonorrhoeae. Int J STD AIDS 2014. [Epub ahead of print]. 